\title{
Medically treated deep neck abscess presenting with occipital headache and meningism
}

\author{
Bon D. Ku $\cdot$ Key Chung Park $\cdot$ Sung Sang Yoon
}

Received: 7 October 2007 / Accepted: 27 November 2007 / Published online: 9 February 2008

(C) Springer-Verlag 2008

\begin{abstract}
We report a 45-year-old man who presented with fever, acute occipital headache, and neck stiffness. He denied immunocompromised state such as diabetes, cancer or AIDS. Lumbar puncture showed normal cerebrospinal fluid findings in spite of laboratory parameters indicating inflammatory reaction. Magnetic resonance imaging of neck demonstrated wide spread enhancing mass of the deep neck space, leading to the final diagnosis of deep neck abscess. A long course of appropriate antibiotic administration finally resolved the inflammation and resulted in a good clinical outcome without surgical drainage. We postulated that deep neck abscess is an important differential diagnosis in a patient with meningism and medical treatment may be available for immunocompetent deep neck abscess.
\end{abstract}

Keywords Meningism - Deep neck abscess ·

Medical treatment

This study was supported by a grant of the Clinical Research Fund of the Myongji Hospital, Kwandong University College of Medicine, GyeongGi, Korea.

\section{B. D. $\mathrm{Ku}(\bowtie)$}

Department of Neurology, Myongji Hospital,

Kwandong University College of Medicine, 697-24,

HwajungDong DukyangGu Goyang, GyeongGi,

South Korea

e-mail: neurodasan@paran.com

K. C. Park · S. S. Yoon

Department of Neurology,

Kyung Hee University College of Medicine,

Seoul, South Korea

\section{Introduction}

Widespread deep neck abscess is an uncommon clinical condition in healthy adults [1]. The main symptoms of deep neck infection are fever and nuchal pain with motion limitation due to soft neck tissue swelling but occipital throbbing headache with meningism is not a common symptom [2]. This type of meningism makes it difficult to diagnose retropharyngeal and deep neck abscess [2, 3]. When infection of the retropharyngeal and deep neck space occurs, usually urgent surgical and antibiotic therapy is required [1]. We describe a case of retropharyngeal and deep neck abscess, which extended anterior to the carotid artery and posterior to the retropharyngeal space resulting in neck pain and stiffness, with successful medical treatment without surgical drainage.

\section{Case report}

A 45-year-old man visited our hospital because of fever, nausea, sore throat, posterior headache, and severe neck pain for 3 days. He complained of stabbing pain radiating from the occipital to parietal area. Coughing and strain exaggerated his headache and neck pain. His nuchal pain was aggravated by neck motion, especially in rotation and extension. So, he could not relax his neck muscle and this provided aggravated immobilization and stiffness.

His previous medical history was unremarkable including immunocompromised states such as diabetes, tuberculosis, malnutrition, and cancer. Prior to the appearance of neck pain, he had 5 days history of flu-like symptoms. He showed stable vital signs except mild fever $\left(37.8^{\circ} \mathrm{C}\right)$ with malaise symptoms. 
In physical and neurological examination, he showed injected throat and neck motion limitation with stiffness and tenderness without neck swelling and redness. Other findings are unremarkable. Blood tests showed leucocytosis $(14,900 / \mu \mathrm{L})$ with $83.2 \%$ neutrophils, C-reactive protein (CRP) of $5.7 \mathrm{mg} / \mathrm{dL}$ and erythrocyte sedimentation ratio of $44 \mathrm{~mm} / \mathrm{h}$, creatine kinase of $790 \mathrm{IU} / \mathrm{L}$. Other laboratory tests including chest X-ray, syphilis screening, human immunodeficiency virus antibody, cancer marker, culture study of blood, sputum and urine were unremarkable. Computed tomography of brain was unremarkable. We performed a lumbar puncture with a tentative diagnosis of meningitis but obtained normal cerebrospinal fluid (CSF) with one white cell per $\mu \mathrm{L}$, zero red blood cells per $\mu \mathrm{L}$, protein of $23 \mathrm{mg} / \mathrm{dL}$, glucose of $75 \mathrm{mg} / \mathrm{dL}$ (blood glucose $109 \mathrm{mg} / \mathrm{dL}$ ).

Then, neck MRI was performed for the exploration of neck pain and stiffness. Neck MRI with gadolinium injection showed a $2.2 \times 4.0 \mathrm{~cm}$ sized ill-defined, inhomogeneous mass in right parapharyngeal and retropharyngeal space with internal, irregular, geographic abscess formation in the nasopharynx and oropharynx level. This mass extended into the ipsilateral carotid space with partial encasement of carotid sheath vessels, longus colli muscle, supraglottic laryngeal wall, and submandibular gland. Also, this mass extended to retropharyngeal and posterior neck space with inhomogeneous enhancement. At the hypopharynx level, there is a displacement and deformity distortion in airway and diffuse retropharyngeal thickening (Fig. 1).

Conservative treatment with appropriate antibiotics was performed instead of surgical drainage by otolaryngology consultation because his clinical condition was stable except for mild fever, occipital headache, and neck stiffness. His clinical symptoms improved gradually and we performed follow-up neck CT after 7 days from the neck MRI. The follow-up neck CT scan showed partially resolved large inflammatory mass in the right parapharyngeal space with small abscess cavities (Fig. 2).

He completed 28 days of antibiotic therapy with penicillin, metronidazole, and roxythromycin. He showed good
Fig. 1 T1-weighted neck magnetic resonance imaging with gadolinium injection showed huge ill-defined inhomogeneous mass in right parapharyngeal and retropharyngeal space with internal, irregular, geographic abscess formation (a, b: arrow). At the hypopharynx level, there is a displacement and deformity distortion in the airway (c: asterisk). This mass anteriorly extended into the ipsilateral carotid space with partial encasement of carotid sheath vessels and related structures (d: arrowhead)
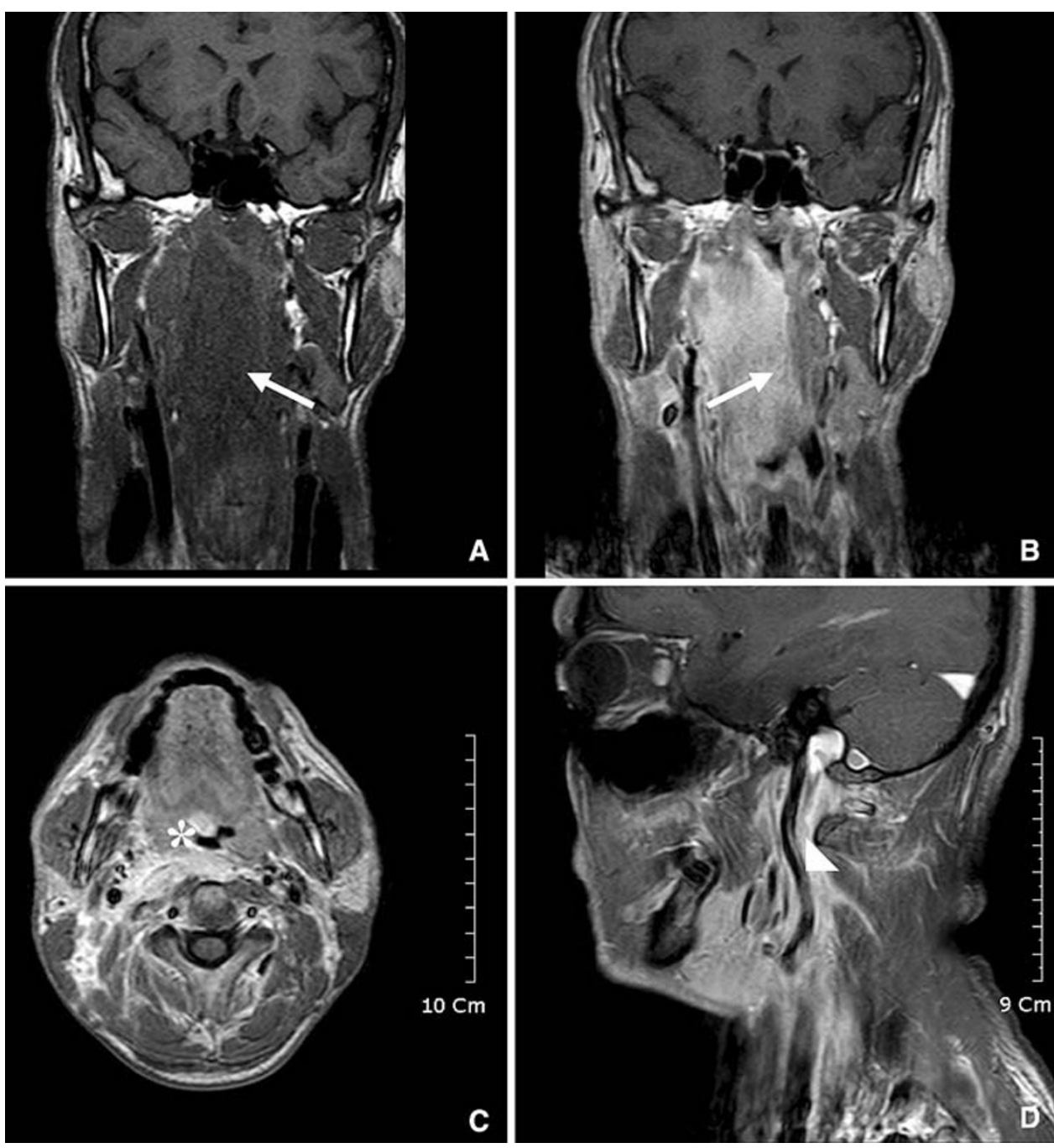
Fig. 2 The follow-up neck computed tomography imaging showed the partially improved large inflammatory mass in the right parapharyngeal space with small abscess cavities (arrow)
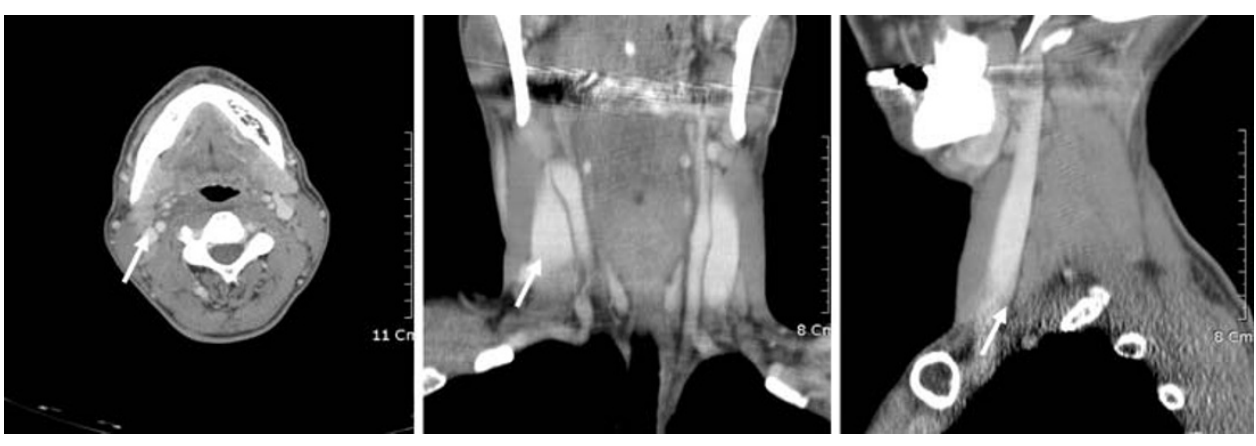

clinical outcome of deep neck infection without headache and neck pain.

\section{Discussion}

Meningeal irritation signs are elicited by inflammation or presence of foreign materials such as blood in the subarachnoid space [4]. Fever and neck stiffness have been described as the classical signs of meningeal irritation, and were considered to be highly specific for meningitis $[4,5]$ However, the presence of meningeal irritation is not highly pathognomonic of meningitis because only $70 \%$ of patients with neck stiffness were confirmed to have meningitis [1, $4,5]$ Meningism has been described in variable conditions such as brain neoplasms, pituitary apoplexy, cervical spondylosis, neck trauma, and specific inflammatory conditions such as cervical osteomyelitis, cervical lymphadenopathy, retropharyngeal abscesses, and severe systemic infection $[2,3,5,6]$.

Some reported cases of deep neck abscess showed concomitant meningeal involvement of retropharyngeal or epidural abscess and were easily misdiagnosed as meningitis for the positive CSF findings [2, 3, 7, 8]. The above-mentioned cases are associated with osteomyelitis, odontogenic infection, cervical spine fracture or epidural abscess and needed urgent surgical exploration. As far as we know, there are two previous reports of retropharyngeal abscess showing meningism without CSF inflammation [5, 9]. But in Rathakrishnan's case, elevated CSF protein $(0.9 \mathrm{~g} / \mathrm{L})$ might contribute to meningeal irritation and in Kielhorn's cases, the abscess are limited to the prevertebral area. However, our patient showed normal CSF profile despite a huge widespread infected mass.

Retropharyngeal abscess in adults is rare because the lymph nodes in that space disappeared in adulthood, and is frequently associated with oral instrumentation, trauma or underlying immunocompromised states such as diabetes, cancer or AIDS [1, 5]. Ventral spread of deep neck abscess could cause a retropharyngeal abscess and dorsal spread would cause epidural abscess and meningitis [3]. In our patient, any predisposing conditions of retropharyngeal abscess were not found, and meningeal and cephalic symptoms were caused by huge inflammation in anterior and posterior neck area due to a contiguous spread of infection.

MRI is thought to be the best modality for diagnosis of abscess formation, infectious granuloma, and odontoid osteomyelitis because of sensitivity to soft tissue changes with good enhancement [3]. In retropharyngeal abscess neck, MRI is an effective diagnostic tool for early accurate diagnosis [5].

The optimal treatment of deep neck abscess is poorly defined. Traditionally, prompt surgical drainage and antibiotics are suggested for the prevention of retropharyngeal abscess rupture leading to meningitis or osteomyelitis cervical spine [6]. In the beginning, we considered surgical drainage. But his clinical condition was gradually improving and he showed no neurological deterioration except neck stiffness. Our patient illustrates the possibility of medical treatment of a retropharyngeal abscess with appropriate antibiotic remedy in an immunocompetent situation. Pages et al. [10] recommended that first-line medical management is required in non-complicated parapharyngeal abscesses with antibiotics and/or steroids. The predisposing factors of complicated deep neck infection are diabetes and more than two involved spaces [11].

In conclusion, we emphasize that retropharyngeal abscesses could give rise to meningism without meningeal involvement and can be treated by appropriate antibiotics in an immunocompetent situation.

\section{References}

1. Haug RH, Picard U, Indresano AT (1990) Diagnosis and treatment of the retropharyngeal abscess in adults. $\mathrm{Br} \mathrm{J}$ Oral Maxillofac Surg 28:34-38

2. Kubo S, Takimoto H, Hosoi K, Toyota S, Karasawa J, Yoshimine $\mathrm{T}$ (2002) Osteomyelitis of the odontoid process associated with meningitis and retropharyngeal abscess-case report. Neurol Med Chir (Tokyo) 42:447-451

3. Tokuoka K, Hamano H, Ohta T, Tamura Y, Shinohara Y (1997) An adult case of purulent meningitis secondary to retropharyngeal and deep neck abscess after treatment of odontogenic infection. Rinsho Shinkeigaku 37:417-419 
4. William WC (2005) DeJong's The neurologic examination, 6th edn. Lippincott Williams \& Wilkins, Philadelphia/Baltimore, pp 617-620

5. Rathakrishnan R, Lim EC, Teoh HL, Chan BP, Seet RC (2005) Retropharyngeal abscess presenting with meningism. Intern Med J 35:567-568

6. Ma RCW, Tsang MW, Ozaki R, Tong PC, Cockram CS (2004) Fever, headache, and a stiff neck. Lancet 363:1868

7. Benoit BG, Russell NA, Cole CW, Clark AJ, McIntyre RW (1983) Meningitis secondary to retropharyngeal abscess. Report of a case occurring in association with cervical spine fracture. Spine 8:438-439

8. Richter S, Reichert N, Roos U, Badent B, Lindner A (2003) Acute headache with meningism and xanthochromic cerebrospinal fluid. An unusual manifestation of cervical epidural abscess. Nervenarzt 74:699-703

9. Kielhorn J, Liebig T, Berlit P (2006) Retropharyngeal inflammation presenting as meningitis: an important differential diagnosis in clinical neurology. Nervenarzt 77:702-705

10. Page C, Biet A, Zaatar R, Strunski V (2007) Parapharyngeal abscess: diagnosis and treatment. Eur Arch Otorhinolaryngol Nov 15 (in press)

11. Lee JK, Kim HD, Lim SC (2007) Predisposing factors of complicated deep neck infection: an analysis of 158 cases. Yonsei Med J 48:55-62 